\title{
Метод расчета взаимной индуктивности системы катушек с использованием модели аксиально намагниченного цилиндра
}

\author{
(C) А.К. Андреев
}

Московский авиационный институт (Национальный исследовательский университет), Москва, Россия

E-mail: alexande_andreev@yahoo.com

Поступило в Редакцию 19 сентября 2019 г.

В окончательной редакции 30 июля 2020 г.

Принято к публикации 30 июля 2020г.

\begin{abstract}
Предложен метод расчета взаимной индуктивности системы катушек с плотной намоткой через энергию аксиально намагниченных цилиндров. Получены формулы для расчета 3D-полей цилиндра и катушек, выраженные через эллиптические интегралы. Показано, что средний по объему размагничивающий фактор цилиндра равен коэффициенту Нагаока для собственной индуктивности коротких катушек.
\end{abstract}

Ключевые слова: взаимная индуктивность, 3D-поля, размагничивающий фактор, коэффициент Нагаока.

DOI: 10.21883/PJTF.2020.21.50198.18042

Методы расчета индуктивности катушек были разработаны Максвеллом (Maxwell, 1873 г.) и Лоренцем (Lorenz, 1879 г.). Обширная информация о методах определения индуктивностей и взаимных индуктивностей, а также ссылки на первоисточники приведены в [1]. На практике при расчетах используются аппроксимирующие формулы либо графоаналитические методы $[1,2]$. В настоящей работе представлен новый альтернативный аналитический метод определения индуктивностей сложной системы катушек. Метод основан на равенстве внутренних энергий катушек и эквивалентных им аксиально намагниченных цилиндров. Задача сводится к расчету энергии и взаимной энергии $n$ цилиндров $E_{n i}$, имитирующих систему катушек. Энергии вычисляются через 3D-поля цилиндров $\mathbf{H}(r, z)=-\Delta \varphi(r, z)$, где $\varphi(r, z)$ - суммарный магнитостатический потенциал торцевых дисковых „зарядов“ цилиндра.

Схема расчета $\varphi(r, z)$ показана на рис. 1 . В скалярной записи связь между $M_{z}, j_{s}$ и $\sigma$ определяется соотношениями [3]:

$$
M_{z}=j_{s}, \quad \sigma=M_{z}
$$

При расчете индуктивностей задаются геометрические параметры катушки: длина $h$, диаметр $2 a$, а также число круговых витков $w$ и ток $I[\mathrm{~A} /$ turn]. Плотность поверхностного тока катушки $j=w I / h$ определяет эквивалентную намагниченность цилиндра $M_{z}=j$ (рис. 1). Влияние неплотности намотки катушки (шага намотки) на корректность модели определено в [4].

При однородной намагниченности $M_{z}$ размагничивающее поле равно

$$
H_{p}(\mathbf{r})=-M_{z} N(\mathbf{r})
$$

где $N(\mathbf{r})$ - размагничивающий фактор, безразмерная величина, зависящая от формы образца $(0 \leqslant N \leqslant 1)$.
$N(\mathbf{r})$ связывает компоненты поля в объеме цилиндра c его намагниченностью: $H_{i}=-N_{i z} M_{z}, i=r, z$. Вне цилиндра $N(\mathbf{r})$ есть коэффициент, по которому рассчитывается поле рассеяния. Размагничивающие факторы $N_{z z}(r, z)$ и $N_{r z}(r, z)$ часто определяются через функции



Рис. 1. Схема расчета $\varphi(r, z) . \mathbf{H}_{p}-$ размагничивающее поле цилиндра, В - индукция цилиндра и катушки, $q\left(r^{\prime}, z^{\prime}\right)-$ точка источника поля, $a(r, z)-$ точка наблюдения, $\mathbf{M}_{z}-$ намагниченность цилиндра, $\mathbf{j}_{s}=\mathbf{M}_{z} \mathbf{n}-$ плотность поверхностного тока катушки, $\sigma=\mathbf{M}_{z} \mathbf{n}-$ плотность торцевых „магнитных зарядов“, $\mathbf{n}$ - вектор нормали к поверхностям цилиндра. 
Энергия и индуктивность магнитных систем

\begin{tabular}{c|c|c|c|c|c|c|c}
\hline $\begin{array}{c}\text { Номер } \\
\text { цилиндра }\end{array}$ & $\begin{array}{c}\text { Радиус } \\
\text { цилиндра } \\
a(n), \mathrm{cm}\end{array}$ & $\begin{array}{c}\text { Энергия } \\
\text { цилиндра } \\
E_{n}, 10^{3} \mathrm{~J}\end{array}$ & $\begin{array}{c}\text { Индуктивность } \\
\text { катушки } \\
L_{n}, 10^{3} \mathrm{H}\end{array}$ & $\begin{array}{c}\text { Размагничи- } \\
\text { вающий } \\
\text { фактор } \bar{N}\end{array}$ & $\begin{array}{c}\text { Коэффициент } \\
\text { Нагаока } \\
k(n)\end{array}$ & $\begin{array}{c}\text { Взаимная } \\
\text { энергия } \\
\text { цилиндров } \\
E_{n i}, 10^{3} \mathrm{~J}\end{array}$ & $\begin{array}{c}\text { Взаимная } \\
\text { индуктивность } \\
\text { катушек } \\
M_{n i}, 10^{4} \mathrm{H}\end{array}$ \\
\hline 1 & 2.5 & 7.175 & 0.574 & 0.128 & 0.872 & $E_{12}=6.542$ & $M_{12}=5.233$ \\
2 & 3.8 & 15 & 1.211 & 0.182 & 0.818 & $E_{13}=6.015$ & $M_{13}=4.811$ \\
3 & 5 & 25 & 2.026 & 0.23 & 0.77 & $E_{23}=0.014$ & $M_{23}=11.04$
\end{tabular}

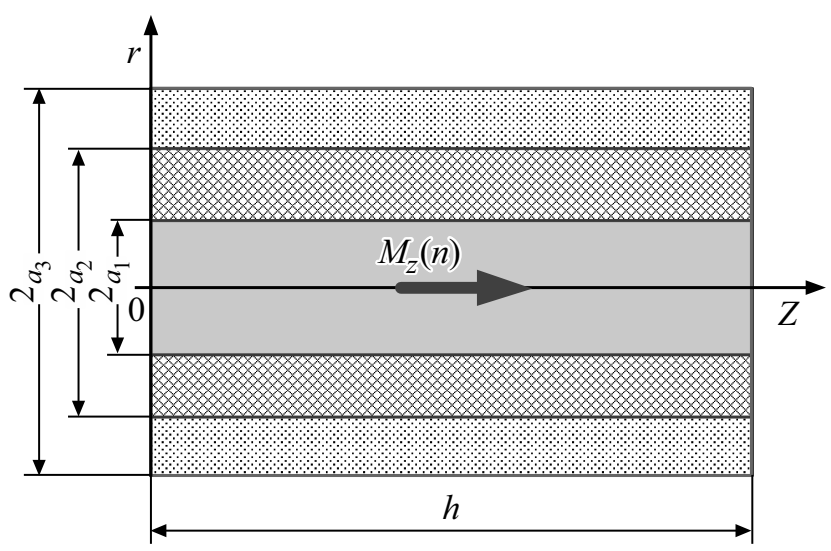

Рис. 2. К расчету взаимной индуктивности. Длины цилиндров $h=15 \mathrm{~cm}$, диаметры цилиндров: $2 a_{1}=5 \mathrm{~cm}, 2 a_{2}=7.6 \mathrm{~cm}$, $2 a_{3}=10 \mathrm{~cm}$.

Бесселя [5]. Однако при использовании этой модели возникают проблемы при численном расчете [6].

Как показано в [7], коэффициенты $N_{z z}(r, z)$ и $N_{r z}(r, z)$ могут быть выражены через эллиптические интегралы. В [8] с учетом [7] определены размагничивающие факторы в объеме цилиндра

$$
\begin{aligned}
& N_{z z}(r, z)=\left[1-\frac{z k_{1} K_{C}\left(k_{1}\right)}{4 \pi \sqrt{a r}}-\frac{\Lambda_{0}\left(\alpha_{1}, \beta_{1}\right)}{4}\right. \\
& \left.-\frac{(h-z) k_{2} K_{C}\left(k_{2}\right)}{4 \pi \sqrt{a r}}-\frac{\Lambda_{0}\left(\alpha_{2}, \beta_{2}\right)}{4}\right], \quad 0 \leqslant z \leqslant h, \quad(3) \\
& N_{r z}(r, z)=\left(\frac{1}{\pi}\right) \sqrt{\frac{a}{r}\left\{\left(\frac{1}{k_{1}}\right)\left[\left(1-\frac{k_{1}^{2}}{2}\right) K_{C}\left(k_{1}\right)-E_{C}\left(k_{1}\right)\right]\right.} \\
& \left.-\left(\frac{1}{k_{2}}\right)\left[\left(1-\frac{k_{2}^{2}}{2}\right) K_{C}\left(k_{2}\right)-E_{C}\left(k_{2}\right)\right]\right\}, \quad 0 \leqslant r \leqslant a,
\end{aligned}
$$

где

$$
K_{C}(k)=\int_{0}^{\pi / 2}\left(1-k^{2} \sin ^{2} \theta\right)^{-1 / 2} d \theta
$$

$$
E_{C}(k)=\int_{0}^{\pi / 2} \sqrt{1-k^{2} \sin ^{2} \theta} d \theta
$$

- полные эллиптические интегралы первого и второго рода с модулями

$$
\begin{gathered}
k_{1}^{2}=4 \operatorname{ar}\left[z^{2}+(a+r)^{2}\right]^{-1} \\
k_{2}^{2}=4 \operatorname{ar}\left[(h-z)^{2}+(a+r)^{2}\right]^{-1} \\
\Lambda_{0}(\alpha, \beta)=\left(\frac{2}{\pi}\right)\left[E_{C}(k) F\left(\beta, \sqrt{1-k^{2}}\right)\right. \\
\left.+K_{C}(k) E\left(\beta, \sqrt{1-k^{2}}\right)-K_{C}(k) F\left(\beta, \sqrt{1-k^{2}}\right)\right]
\end{gathered}
$$

- лямбда-функции Хеймана, полные эллиптические интегралы третьего рода [9],

$$
\begin{gathered}
F(\beta, m)=\int_{0}^{\beta}\left(1-m^{2} \sin ^{2} \theta\right)^{-1 / 2} d \theta, \\
E(\beta, m)=\int_{0}^{\beta} \sqrt{1-m^{2} \sin ^{2} \theta} d \theta
\end{gathered}
$$

- неполные эллиптические интегралы первого и второго рода с дополнительными модулями $m$ и амплитудами $\beta$ :

$$
\begin{gathered}
m_{1}=\sqrt{1-k_{1}^{2}}, \quad m_{2}=\sqrt{1-k_{2}^{2}}, \\
\beta_{1}=\arcsin \left[z\left[z^{2}+(a-r)^{2}\right]^{-1 / 2}\right], \\
\beta_{2}=\arcsin \left[(h-z)\left[(h-z)^{2}+(a-r)^{2}\right]^{-1 / 2}\right],
\end{gathered}
$$

где $\beta_{1}, \beta_{2}$ - главные значения $\arcsin$.

При расчетах полей вне цилиндрической области полей рассеяния - необходимо переопределение формул (3), (4). Далее получены коэффициенты $N_{z z}$ и $N_{r z}$, позволяющие вычислять 3D-поля во всем пространстве. Формулы записаны раздельно для характерных областей 
внутри и вне цилиндра в пределах диапазонов изменения $z$ и $r[6]$ :

$$
N_{z z}(r, z)=\left\{\begin{array}{l}
{\left[1-\frac{z k_{1} K_{C}\left(k_{1}\right)}{4 \pi \sqrt{a r}}-\frac{\Lambda_{0}\left(\alpha_{1}, \beta_{1}\right)}{4}-\frac{(h-z) k_{2} K_{C}\left(k_{2}\right)}{4 \pi \sqrt{a r}}-\frac{\Lambda_{0}\left(\alpha_{2}, \beta_{2}\right)}{4}\right], \text { если }(0 \leqslant z \leqslant h) \wedge(|r| \leqslant a)} \\
0, \text { если }(0 \leqslant z \leqslant h) \wedge(r=0), \\
-\frac{z k_{1} K_{C}\left(k_{1}\right)}{4 \pi \sqrt{a r}}+\frac{\Lambda_{0}\left(\alpha_{1}, \beta_{1}\right)}{4}-\frac{(h-z) k_{2} K_{C}\left(k_{2}\right)}{4 \pi \sqrt{a r}}-\frac{\Lambda_{0}\left(\alpha_{2}, \beta_{2}\right)}{4}, \text { если }(z<0) \wedge(0<|r|)<a, \\
-\frac{z k_{1} K_{C}\left(k_{1}\right)}{4 \pi \sqrt{a r}}-\frac{\Lambda_{0}\left(\alpha_{1}, \beta_{1}\right)}{4}-\frac{(h-z) k_{2} K_{C}\left(k_{2}\right)}{4 \pi \sqrt{a r}}+\frac{\Lambda_{0}\left(\alpha_{2}, \beta_{2}\right)}{4}, \text { если }(z>h) \wedge(0<|r|)<a, \\
-\frac{z k_{1} K_{C}\left(k_{1}\right)}{4 \pi \sqrt{a r}}+\frac{\Lambda_{0}\left(\alpha_{1}, \beta_{1}\right)}{4}-\frac{(h-z) k_{2} K_{C}\left(k_{2}\right)}{4 \pi \sqrt{a r}}+\frac{\Lambda_{0}\left(\alpha_{2}, \beta_{2}\right)}{4}, \text { если }(0<z<h) \wedge(|r|>a), \\
-\frac{z k_{1} K_{C}\left(k_{1}\right)}{4 \pi \sqrt{a r}}-\frac{\Lambda_{0}\left(\alpha_{1}, \beta_{1}\right)}{4}-\frac{(h-z) k_{2} K_{C}\left(k_{2}\right)}{4 \pi \sqrt{a r}}+\frac{\Lambda_{0}\left(\alpha_{2}, \beta_{2}\right)}{4}, \text { если }(z<0) \wedge(|r|>a), \\
-\frac{z k_{1} K_{C}\left(k_{1}\right)}{4 \pi \sqrt{a r}}+\frac{\Lambda_{0}\left(\alpha_{1}, \beta_{1}\right)}{4}-\frac{(h-z) k_{2} K_{C}\left(k_{2}\right)}{4 \pi \sqrt{a r}}-\frac{\Lambda_{0}\left(\alpha_{2}, \beta_{2}\right)}{4}, \text { если }(z>h) \wedge(|r|>a), \\
-\frac{1}{2}\left[\frac{h-z}{\sqrt{(h-z)^{2}}+a^{2}}+\frac{z}{\sqrt{z^{2}+a^{2}}}-\frac{h-z}{|h-z|}-\frac{z}{|z|}\right], \text { если } r=0,
\end{array}\right.
$$

0 везде,

$$
N_{r z}(r, z)=\left\{\begin{aligned}
&\left(\frac{1}{\pi}\right)\left(\sqrt{\frac{a}{r}}\right)\left\{\left(\frac{1}{k_{1}}\right)\left[\left(1-\frac{k_{1}^{2}}{2}\right) K_{C}\left(k_{1}\right)-E_{C}\left(k_{1}\right)\right]\right.\left.-\left(\frac{1}{k_{2}}\right)\left[\left(1-\frac{k_{2}^{2}}{2}\right) K_{C}\left(k_{2}\right)-E_{C}\left(k_{2}\right)\right]\right\}, \text { если } r>0, \\
&-\left(\frac{1}{\pi}\right)\left(\sqrt{\frac{a}{r}}\right)\left\{\left(\frac{1}{k_{1}}\right)\left[\left(1-\frac{k_{1}^{2}}{2}\right) K_{C}\left(k_{1}\right)-E_{C}\left(k_{1}\right)\right]\right. \\
&\left.-\left(\frac{1}{k_{2}}\right)\left[\left(1-\frac{k_{2}^{2}}{2}\right) K_{C}\left(k_{2}\right)-E_{C}\left(k_{2}\right)\right]\right\}, \text { если } r<0, \\
& \text { 0 везде. }
\end{aligned}\right.
$$

Последняя строка в (5) определяет размагничивающий фактор на оси. С учетом (2) $z$ - и $r$-компоненты поля и индукции цилиндра равны

$$
\begin{gathered}
H_{z}(r, z)=-M_{z} N_{z z}(r, z), \\
H_{r}(r, z)=-M_{z} N_{r z}(r, z), \\
B_{z}(r, z)=\mid \begin{array}{l}
\mu_{0}\left(H_{z}+M_{z}\right), \text { если }(0 \leqslant z \leqslant h) \wedge(0 \leqslant|r| \leqslant a), \\
\mu_{0} H_{z}(r, z) \text { везде, }
\end{array} \\
B_{r}(r, z)=\mu_{0} H_{r}(r, z) .
\end{gathered}
$$

Расчет полей рассеяния через эллиптические интегралы обеспечивает получение стабильных численных результатов при любых параметрах магнитной системы. В модели не накладываются какие-либо ограничения на взаимное расположение цилиндров (катушек) и их геометрические и магнитные параметры. Программы расчета $N_{z z}(r, z)$ и $N_{r z}(r, z)$ опубликованы в $[10]$. В $[11,12]$ получены аналитические выражения для полей цилиндра при произвольной ориентации намагниченности.

Далее приведена схема расчета взаимной индуктивности в системе трех соосных катушек $(n=3)$. На рис. 2 представлены конфигурация системы и используемые расчетные параметры. Равные намагниченности и длины цилиндров были введены для демонстрации работоспособности модели на простом примере. Расчеты выполнены в соответствии с формулами (5), (9). Суть метода заключается в расчете взаимной энергии между всеми парами цилиндров. Понятие „индуктивность цилиндра“ не имеет физического смысла. Вычисление же энергии цилиндра есть важнейший промежуточный 
этап при расчете взаимной индуктивности катушек. Цилиндры нумеруются с присвоением номера (индекса) для каждого параметра и формулы. Задавая равные токи в моделируемых катушках $I(n)=5 \mathrm{~A} /$ turn, получаем $M_{z}(n)=6.667 \mathrm{~A} / \mathrm{m}$. Энергия $n$-го цилиндра радиусом $a(n)$ в поле $i$-го цилиндра радиусом $a(i)$ равна

$$
E_{c y l}(n, i)=2 \pi \int_{0}^{h} \int_{0}^{a(n)} \frac{M_{z}(n) B_{z}(i, r, z)}{2} r d r d z .
$$

Коэффициент взаимной индуктивности с учетом (11) определяется по формуле

$$
M_{n i}=\frac{2 E_{c y l}(n, i)}{I(n) I(i)} .
$$

Учитывая равенство взаимных энергий цилиндров, расчеты $M_{n i}$ достаточно проводить только для одной пары системы. Энергию $n$-го цилиндра и собственную индуктивность $n$-й катушки $L$ вычисляем по формулам (11), (12), полагая $n=i$.

Собственная индуктивность катушки $L$ определяется также через коэффициент Нагаока $k 1_{L}$ [13] с помощью соотношения

$$
L=\left(\mu_{0} w^{2} \pi(2 a)^{2} / 4 h\right) k 1_{L} \quad\left(0 \leqslant k_{L} \leqslant 1\right) .
$$

Выражение в скобках есть индуктивность участка длиной $h$ бесконечно длинного соленоида. Из (5) (строки 1,7$)$ аналог $k 1_{L}$ следует из среднего по объему размагничивающего фактора цилиндра $\bar{N}_{z}$ :

$$
k_{L}=1-\frac{2 \pi}{\pi a^{2} h} \int_{0}^{h} \int_{0}^{a} N_{z z}(r, z) d r d z=1-\bar{N}_{z} .
$$

Результаты расчетов параметров магнитных систем при $n=3$ суммированы в таблице.

Тестовые расчеты $M_{n i}$ основных схем взаимного расположения катушек по приведенной модели соответствуют результатам, полученным в [2].

Независимость размагничивающих факторов цилиндров от внешних полей позволяет применять ,энергетический подход“ для расчета сложных систем, составленных из отдельных элементов. Существуют и другие области применения полученных в настоящей работе результатов. Например, по известной взаимной энергии цилиндров можно определить пондеромоторные силы в системе катушек, минуя стадию расчета индуктивности.

\section{Список литературы}

[1] http: //coil32.ru/; http:/g3ynh.info/zdocs/magnetics/

[2] Калантаров П.Л., Цейтлин Л.А. Расчет индуктивностей. Л.: Энергоатомиздат, 1986. $488 \mathrm{c}$.

[3] Парселл Э. Электричество и магнетизм. М.: Наука, 1975. $440 \mathrm{c}$.

[4] Андреев А.К. // Проблемы машиностроения и надежности машин. 2019. № 1. С. 34-43.

[5] Bateman H. Partial differential equations of mathematical physics. N.Y.: Dover Publ., 1944. 556 p.

[6] Андреев А.К. Магнитостатика ферромагнетиков. М.: Изд-во МАИ, 2011. $162 \mathrm{c.}$

[7] Eason G., Noble B., Sneddon I.N. // Phil. Trans. Roy. Soc. Lond. A. 1955. V. 247. P. 529-551.

[8] Joseph R.I., Schlömann E. // J. Appl. Phys. 1964. V. 36. P. $1579-1593$.

[9] Справочник по специальным функциям / Под ред. М. Абрамовица, И. Стиган. М.: Наука, 1979. 832 с.

[10] Андреев А.К. Программы для ЭВМ. Свидетельство о госрегистрации № 2012614673. 2012. Бюл. № 3. Ч. 2. C. 378-379.

[11] Taniguchi T. // J. Magn. Magn. Mater. 2018. V. 452. P. 464472.

[12] Caciagli A., Baars R.J., Philipse A.P., Kuipers B.W.M. // J. Magn. Magn. Mater. 2018. V. 456. P. 423-432.

[13] Nagaoka H. // J. Coll. Sci. 1909. V. 27. P. 18-33.

\section{Конфликт интересов}

Автор заявляет, что у него нет конфликта интересов. 